\title{
Contrasting dynamics and environmental controls of dispersed bacteria along a hydrologic gradient
}

\author{
Clara Ruiz-González, ${ }^{1 *}$ Juan Pablo Niño-García, ${ }^{2}$ Martin Berggren, ${ }^{3}$ Paul A. del Giorgio ${ }^{4}$ \\ ${ }^{1}$ Institut de Ciències del Mar (ICM-CSIC), Passeig Marítim de la Barceloneta 37-49, 08003, Barcelona, Spain; ${ }^{2}$ Escuela de Microbiología, \\ Universidad de Antioquia, Ciudad Universitaria Calle 67 No 53-108, Medellín, Colombia; ${ }^{3}$ Department of Physical Geography and \\ Ecosystem Science, Lund University, Sölvegatan 12, SE-223 62, Lund, Sweden; ${ }^{4}$ Groupe de Recherche Interuniversitaire en Limnologie \\ et en Environnement Aquatique (GRIL), Département des Sciences Biologiques, Université du Québec à Montréal, Case Postale 8888, \\ succursale Centre-Ville, Montréal, QC, H3C 3P8, Canada \\ *Corresponding author: clara.ruiz.glez@gmail.com
}

\begin{abstract}
Freshwater bacterioplankton communities are influenced by the transport of bacteria from the surrounding terrestrial environments. It has been shown that, although most of these dispersed bacteria gradually disappear along the hydrologic continuum, some can thrive in aquatic systems and become dominant, leading to a gradual succession of communities. Here we aimed at exploring the environmental factors driving the structure of such contrasting bacterial populations as well as their functional properties. Using Illumina sequencing of the 16S rRNA gene, we characterized the taxonomic composition of bacterioplankton communities from 10 streams and rivers in Québec spanning the whole hydrologic continuum (river Strahler order 0 to 7), which were sampled in two occasions. With the aim to understand the fate and controls of the transported bacteria, among the taxa present at the origin of the hydrologic gradient (i.e., in the smallest headwater streams) we identified two types of dynamics: i) 'Tourist' taxa, which were those that decreased in abundance from the headwaters towards the largest rivers, and ii) 'Seed' taxa, those that increased their abundances along the hydrologic continuum. Communities changed gradually from the fast-flowing headwater streams dominated by 'Tourist' taxa (ca. 95\% of the sequences) towards the largest rivers (Strahler order 4-7) where 'Seed' taxa comprised up to $80 \%$ of community sequences. Variation in taxonomic composition of the communities dominated by 'Tourist' taxa in streams seemed related to different degree of terrestrial inputs, whereas compositional changes in 'Seed' communities in the large rivers were linked to differences in autochthonous processes. Finally, the two types of communities differed significantly in their metabolic potential assessed through Biolog Ecoplates. All this suggests that hydrologic transport modulates the gradual replacement of two contrasting population types subjected to different environmental controls and with different metabolic potentials. Moreover, we show that the separate exploration of the two pools of taxa allows unveiling environmental drivers and processes operating on them that remain hidden if explored at the whole community level.
\end{abstract}

Key words: Dispersal; river continuum; environmental sorting; aquatic bacterial communities; Illumina sequencing.

Received: December 2017. Accepted: December 2017.

\section{INTRODUCTION}

Freshwater ecosystems are tightly linked to the surrounding terrestrial landscape, which continuously exports soil material and microorganisms to the water. Understanding the functioning and structuring of lake and river bacterioplankton communities therefore requires taking into consideration these potential linkages between habitats. However, most current work on microbial biogeography is restricted to single types of ecosystems (i.e., only lakes, only soils, only rivers), thus disregarding the potential dispersal of taxa between communities from similar or different habitats, and rendering a rather fragmented view of microbial biogeography.

The few recent studies considering such land-water linkages have shown not only that this connectivity with the terrestrial environment may influence aquatic microbial communities by providing nutrients and dissolved or- ganic matter of different quality (Besemer et al., 2013; Berggren and del Giorgio, 2015; Ruiz-González et al., 2015a; Wilhelm et al., 2015), but also that the immigration of microbes from the surrounding catchment can largely explain the local composition of the receiving community. For example, terrestrial runoff causes the advection of high numbers of bacterial taxa to aquatic bodies, and consequently the systems most tightly connected to the landscape will be more strongly influenced by this transport of terrestrial bacteria. Although headwater streams have been suggested to act as integrators and vectors of microbial diversity from soil sources (Crump et al., 2007; Nelson et al., 2009; Besemer et al., 2012, 2013), only a few studies have directly explored this (Savio et al 2015; Ruiz-González et al., 2015a; Niño-García et al 2016a), showing that most of these dispersed bacteria, which dominate communities in headwaters, gradually disappear along the river continuum. This results in a di- 
rectional structuring of aquatic communities and a gradual decrease in taxonomic richness that has been observed both along individual rivers (Savio et al 2015) or across multiple unconnected aquatic sites (Ruiz-González et al., 2015a; Niño-García et al 2016a). More importantly, however, some of these transported soil bacteria seem to be able to grow during transit in the water and to dominate freshwater bacterioplankton communities when the water residence time is long enough, i.e., in large rivers and lakes (Crump et al 2012, Ruiz-González et al., 2015a).

The residence time and the distance travelled by a given parcel of water will thus determine the relative proportions of these two contrasting pools of communities that move along the aquatic continuum. The most basic distinction is between the ones that thrive and adapt to local conditions (i.e., 'seed' taxa, sensu Ruiz-González et al., 2015a) and those that are mal-adapted to the aquatic environment and which therefore decrease in abundance along the hydrologic gradient due to death or dilution (i.e., 'tourist' taxa, sensu Newton et al., 2011). In support of this idea, several studies have highlighted the role of hydrology, and water residence time in particular, as a major driver of the assembly of aquatic communities in freshwater systems. For example, it was shown that the difference between bacterioplankton communities in lakes and the immigrant assemblages arriving through their inlets varies as a function of lake water residence time (Lindström and Bergström 2004; Lindström et al., 2006). In this regard, Crump et al. (2007) suggested that bacteria transported by advection from upstream sources should constitute a small fraction of the total cells in systems with water residence times longer than bacterial doubling times. Other studies have explicitly explored the dynamics of different groups of bacterial taxa, showing that, whereas communities at the origin of the hydrologic continuum (i.e., in the smallest headwater streams) are mostly dominated by taxa washed from soils that likely cannot thrive in aquatic systems, downstream systems with longer water residence times mostly comprise typical freshwater taxa adapted to local conditions (Savio et al., 2015; Ruiz-González et al., 2015a; Niño-García et al., 2016a). Related to this, Niño-García et al., (2016a) found that, although $\mathrm{pH}$ seemed to shape bacterial communities along the whole aquatic continuum in the boreal landscape (ca. 400 streams, rivers and lakes), its role as a driver of taxonomic differences was much more important in large rivers and lakes than in headwater streams where water residence times are shorter. The observed response to $\mathrm{pH}$ in fast-flowing streams was interpreted as a signature of the landscape past environmental conditions (Niño-García et al., 2016a) rather than to in-stream selection. Since this was done considering the overall communities, which harbour both types of populations, it is possible that more clear links between environmental drivers and taxonomy will appear considering the two populations separately. For example, catchment properties should be more important in shaping the observed taxonomic composition of 'Tourist' taxa in headwater streams communities (Lear et al., 2013), whereas communities from large rivers or lakes should be mostly structured by local aquatic conditions (Logares et al., 2013; Souffreau et al., 2015).

The corollary of the above is that the succession and replacement of populations with increasing water residence time should be accompanied by shifts in the functional capacities of the communities, since the selection and growth of 'Seed' taxa along the aquatic continuum should be mostly driven by environmental factors that require a specific functional response. This link between the functional and taxonomic successions that occur along the aquatic continuum may be blurred by the presence of a large fraction of 'Tourist' taxa that do not necessarily contribute to community functioning. In this regard, NiñoGarcía et al. (2016b) showed that the presence of most rare taxa across lake bacterioplankton assemblages was due to hydrologic transport from the associated rivers and, although they may not be functional, they comprised most of the species richness detected across lakes. This may explain why, in a previous study where we explored the large-scale spatial patterns in the metabolic profiles of boreal lake and river bacterioplankton communities (RuizGonzález et al., 2015b), we found little or no coupling between changes in structure and function. This of course has major implications on our capacity to predict how variations in the observed taxonomic composition may impact community metabolism or their responses to environmental perturbations, and calls for the need to partition assemblages into components that are subjected to different controls, in order to explore the links between the environment, taxonomic identity, and community functioning in these freshwater microbial assemblages.

Here we aimed at exploring the fate, the environmental drivers and the metabolic profiles of the dispersed bacterial populations along a hydrologic gradient. To do so, using Illumina sequencing of the 16S rRNA gene, we assessed the taxonomic composition of bacterioplankton communities inhabiting 10 different rivers from two temperate regions in Québec, ranging from small headwater streams (Strahler order 0 or 1) to large rivers (Strahler order 7). The selected rivers flow through very different landscapes, including forested areas, wetlands and scrublands (Berggren and del Giorgio, 2015), which had been previously shown to influence the patterns of microbial metabolism in these same rivers (Berggren and del Giorgio, 2015). We searched for two types of dynamics among headwater bacterial taxa: i) those that decreased in abundance along the hydrologic gradient (i.e., 'Tourist' taxa, sensu Newton et al., 2011), which thus represent taxa that 
may not be able to thrive in downstream waters, and ii) those that increased in abundance likely due to in-river selection and growth (i.e., 'Seed' taxa, sensu RuizGonzález et al., 2015a). In order to understand the ecological underpinnings of the two populations, we explored the environmental variables explaining compositional changes of these communities at both extremes of the hydrologic gradient (i.e., where they were dominant), as well as their metabolic potential using Biolog Ecoplates. We hypothesized that there should also be a watershed-specific signature in the taxonomic composition of the communities dominated by 'Tourist' taxa, which reflect the broad catchment differences, whereas assemblages dominated by 'Seed' taxa should reflect in-river physicochemical conditions.

\section{METHODS}

\section{Study sites, sampling and basic parameters}

The sampling design has been previously described in Berggren and del Giorgio (2015). Briefly, we sampled 10 streams and rivers for the characterization of bacterioplankton communities with Illumina sequencing (Illumina Inc., San Diego, CA, USA) of the 16S rRNA gene. The streams were located in two regions of Quèbec, Canada, and spanned the whole hydrologic gradient ranging from small headwater streams of Strahler order 0 or 1 to large rivers (order 6 or 7); they were also selected to maximize the differences in catchment type (peat bogs versus a range of forest soils) and total upstream distance of each site (Tab. 1). Additional details on the environmental characteristics of the sites can be found in Tab. 1 of Berggren and del Giorgio (2015). Each site was sampled twice between 31 May and 24 August 2010, during late spring and summer low-flow conditions, in order to assess the consistency of the patterns observed. Two of the rivers could be sampled only once (Tab. 1).
Water was collected at depths of $20-30 \mathrm{~cm}$ and all samples were stored in cooling boxes in the dark until analysis. Water temperature, dissolved oxygen (DO), $\mathrm{pH}$ and conductivity were measured in situ with an YSI probe. At each site, a water sub-sample was filtered in situ through $0.45 \mu \mathrm{m}$ (PES cartridge, Sarstedt) and stored in acid-washed glass vials for dissolved organic carbon (DOC), nutrients and optical analyses, and another sample was kept in acid-rinsed bottles for DNA processing in the laboratory and to measure the substrate utilization profiles using Biolog Ecoplates ${ }^{\mathrm{TM}}$ (see below).

DOC concentration was measured on an OI1010 TOC analyser. The optical properties of DOC were measured as indices of its composition and source. Coloured dissolved organic matter (cDOM) was quantified as the absorbance at $440 \mathrm{~nm}$ using an Ultrospec 3100 spectrophotometer. DOC composition was described on the basis of fluorescence absorption/emission spectra (EEMS) measured in a Shimadzu RF5301 PC spectrofluorophotometer across excitation/emission wave- lengths of 275-450 nm and 280$600 \mathrm{~nm}$, respectively. Six main fluorescence components were recovered from the EEMS using parallel factor analysis (PARAFAC): Components 1 to 3 (C1-C3), previously related to refractory, humic material of presumably terrestrial origin, and C4-C6, associated with more biolabile freshly produced DOM (for details see Lapierre and del Giorgio, 2014; Stubbins et al., 2014). C5 and C6 have been linked to autochthonous processes, having C6 a fluorescence signature typical of protein-like material. The percentage contribution of each component was calculated relative to the total fluorescence of the six PARAFAC components.

\section{Geographical analyses}

River length, total upstream distance and Strahler order, catchment areas and elevation of the sampled sites were obtained using the ARCMAP 10 and ARCGIS V10

Tab. 1. Characteristics of the different sampling sites ordered based on their distance to their respective headwaters. Most rivers were sampled in two occasions, but two sites were sampled only once.

\begin{tabular}{|c|c|c|c|c|c|}
\hline Name & Region & Season & Strahler river order & Upstream distance (m) & Catchment type \\
\hline Stream \# 09 & Abitibi & Spring/summer & 0 & 454 & Broad-leaf forest \\
\hline Stream \# 08 & Abitibi & Spring/summer & 1 & 1193 & Broad-leaf forest \\
\hline Stream \# 07 & Abitibi & Spring/summer & 1 & 1828 & Broad-leaf forest \\
\hline Petit Nord Suivit & Baie James & Spring/summer & 2 & 2205 & Bog \\
\hline Petit Aiguebelle Suivit & Abitibi & Spring/summer & 2 & 2951 & Mixed forest \\
\hline Ruisseau Brunet & Abitibi & Spring/summer & 4 & 7149 & Mixed forest \\
\hline Inter-Nord Suivit & Baie James & Spring & 4 & 19575 & Bog \\
\hline Magousi & Abitibi & Summer & 6 & 66488 & Clay soils \\
\hline Kinojevis & Abitibi & Spring/summer & 7 & 172271 & Clay soils \\
\hline Harricana & Baie James & Spring/summer & 7 & 232654 & Bog \\
\hline
\end{tabular}


software (ESRI Inc., Redland, CA, USA) applied on Digital Elevation Models (DEM) derived from (1:50 000) maps. For each catchment, we averaged slope based on DEM, and determined various landcover properties from Geobase (2009).

\section{Bacterial community taxonomic composition}

Bacterial biomass was collected filtering $300-500 \mathrm{~mL}$ of water through $0.22 \mathrm{~mm}$ (47 mm, GSWP, Millipore). Bacterial DNA was extracted using the MoBio (Carlsbad, CA, USA) DNA extraction kit, following the manufacturer's instructions. Tagged amplicons of the 16SrRNA gene (V3-V4 region) were obtained with the primers 515F and 806R using a two-step PCR and the Access Array barcode library (Fluidigm), and sequenced on an Illumina MiSeq2000 using a paired-end approach (Caporaso et al., 2012). Paired-end reads were assembled with FLASH (Magoc and Salzberg, 2011) and sequences between 250 and $290 \mathrm{bp}$ were used for downstream analyses in QIIME to remove primers, low-quality, archaeal and chloroplast reads (Caporaso et al., 2010). Quality sequences were binned into operational taxonomic units (OTUs, $\geq 97 \%$ similarity) using UCLUST v1.22q (Edgar, 2010) and RDP classifier (Wang et al., 2007). Representative sequences were then aligned against the SILVAv108 reference alignment (Pruesse et al., 2007). To ensure that rare bacteria were not the result of sequencing errors, we discarded all OTUs showing $<4$ sequences. To enable comparisons between samples, the OTU table was randomly subsampled to ensure an equal number of sequences per sample, based on the sample with the least number of reads $(72,798 \mathrm{se}-$ quences).

\section{Identification of 'Seed' and 'Tourist' OTUs}

In order to explore the dynamics of the taxa dispersed along the hydrologic continuum, we first selected all the OTUs present in the smallest streams of Strahler order 0 (those that did not appear in topographic maps because they are too small or temporary) or order 1. Among those, we further categorized OTUs as i) 'Tourists' (sensu Newton et al., 2011), if their abundances were higher in streams than in the largest rivers of order 6 or 7 (which may represent terrestrial taxa washed from soils that cannot thrive in aquatic conditions), or ii) 'Seeds', if their abundances were higher in the largest rivers than in headwaters of order 0 and 1 (and thus might represent taxa seeding aquatic communities; Ruiz-González et al., 2015a).

\section{Bacterial substrate utilization patterns}

The capacity of the riverine bacterial communities to respire 31 carbon substrates was assessed using the Biolog Ecoplate $^{\mathrm{TM}}$ (CA, USA) as detailed in Berggren and del Giorgio (2015). Briefly, one plate per site was incubated in the dark and at $20^{\circ} \mathrm{C}$, and the absorbance was measured periodically (every 6 or 12 hours depending on colour development rates) until an asymptote was reached, between 2 and 9 days. At each time point, the overall colour development of the plates was expressed as the average well colour development (AWCD), and the mean colour development of each compound was calculated as the blankcorrected mean absorbance of each substrate measured at the time when the AWCD was closest to 0.5 (Garland et al., 2001), usually between days 1 and 4. All Biolog incubations were initiated 2-6 h after field sampling.

\section{Statistical analyses}

Differences in bacterial taxonomic composition between ecosystem types were tested with ANOSIM (Clarke, 1993). The Shannon index was calculated as an estimate of bacterial taxonomic diversity. Bray-Curtis distance was used as an estimator of taxonomic dissimilarity between samples, and was visualised by non-metric multidimensional scaling (NMDS) analysis. We performed least squares linear regression to establish relationships between individual variables. All analyses were performed in JMP 9.0.1 (SAS Institute, NC, USA) or R 3.0.0 software (R Core Team, 2013; Vegan package).

\section{RESULTS}

After rarefaction, sequencing of the 16S rRNA gene from the 18 samples resulted in $1,310,364$ quality sequences, which clustered into 64,489 OTUs. Altogether, 59 bacterial phyla were detected, yet sequences were mainly dominated by the phylum Proteobacteria ( $45 \%$ of the total number of sequences), followed by Actinobacteria (14\%), Bacteroidetes (12\%), and OD1 (7\%) and Verrucomicrobia (4\%). The number of OTUs per site ranged from 4376 to 17604 , and we observed pronounced decreases in OTU richness and taxonomic diversity (i.e., Shannon index) along a gradient of increasing distance from the headwaters (Fig. 1 a,b). Interestingly, this pattern was also reflected in a clear structuring of communities depending on the distance travelled (Fig. 1f) regardless of the season, region, or catchment type (Figs $1 \mathrm{c}-\mathrm{e}$ ).

In order to explore the spatial dynamics of the taxa dispersed along the hydrologic continuum, we identified, among the OTUs present at the origin of the hydrologic gradient (i.e., in the smallest headwaters), two groups of taxa: 'Tourists', if their abundances decreased from headwaters to large rivers, or 'Seeds' if their abundances increased towards downstream waters (see Methods). We assume that whereas the former represent taxa washed from soils that cannot thrive in the aquatic environment, the latter comprise taxa (presumably also washed from soils, see Ruiz-González et al., 2015a) able to thrive in the aquatic 
environment. Doing so, 38,725 OTUs were categorized as 'Tourists', and only 6026 as 'Seeds' (Fig. 2). Together, these two groups represented $92.4 \%$ of the total sequences in the dataset. The remaining taxa, 19,738 OTUs, could not be clearly categorized in any of the two groups due to extreme rareness and lack of any discernible spatial pattern, but these uncategorized taxa represented a very small fraction of the total sequences (7.4\%), meaning that these simple criteria recovered most of the diversity across the sampling sites.

We observed clear contrasting patterns between the two pools of taxa (Fig. 2): 'Tourist' OTUs largely dominated headwater communities in all cases but showed pronounced decreases towards the largest rivers (Fig. 2a), whereas 'Seed' OTUs shifted from making up less than $9 \%$ of headwater sequences to numerically dominate the largest rivers (ca. $80 \%$ of sequences in rivers of order 6 and 7, Fig. 2b). The decrease in 'Tourist' OTU abundance was clearly due to a comparable reduction in the number of OTUs (Fig. 2c), but the dominance of 'Seeds' in downstream ecosystems seemed caused by large increases in abundance of a small number of OTUs dispersed from the headwaters (Fig. 2d). Interestingly, in both cases we observed that such shifts in abundance stabilized after a cumulative distance of around $7 \mathrm{~km}$ from the headwaters, which corresponded to rivers of Strahler order 4 (Fig. 2). Beyond that point, both the 'Tourist' and 'Seed' OTUs did not show further changes in relative abundance regardless of the very large differences in the total upstream distance.

In terms of the taxonomic composition, the two taxa pools differed largely from each other (Fig. 3), and whereas downstream rivers were numerically dominated by groups like Betaproteobacteria, Actinobacteria and Cyanobacteria, in headwater streams we found higher abundances of Alpha-, Delta- and Gammaproteobacteria, OD1 or OP3, among others (Fig. 3). Interestingly, this distribution of taxonomic groups was remarkably similar between the two sampling occasions.

When the two taxa pools were visualized separately in an NMDS, we found that in both cases the communities were also structured following the patterns as a function of distance from the headwaters described above for the whole community (Fig. 4), and again this pattern was independent of the season, region or catchment type (details not shown). Interestingly, moreover, we observed that, in those sites where either 'Tourist' or 'Seed' OTUs were most abundant, we could detect two different groups of communities based on their taxonomic composition ( $\mathrm{t} 1$ and $\mathrm{t} 2$ in 'Tourist'-dominated communities and s1 and s2 in 'Seed'-dominated communities, see arrows in Fig. 4).
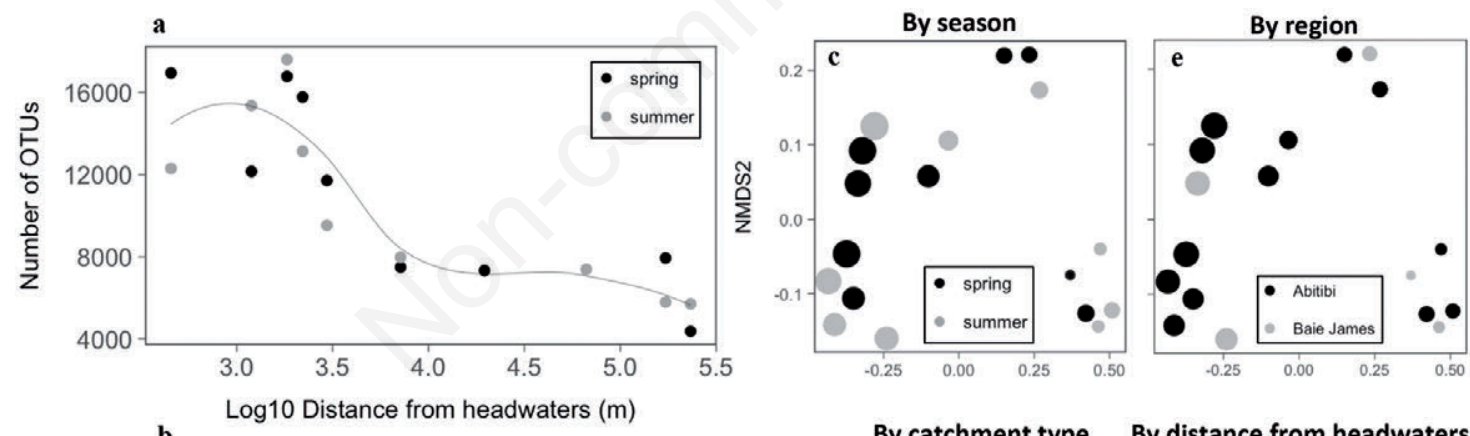

OTU richness
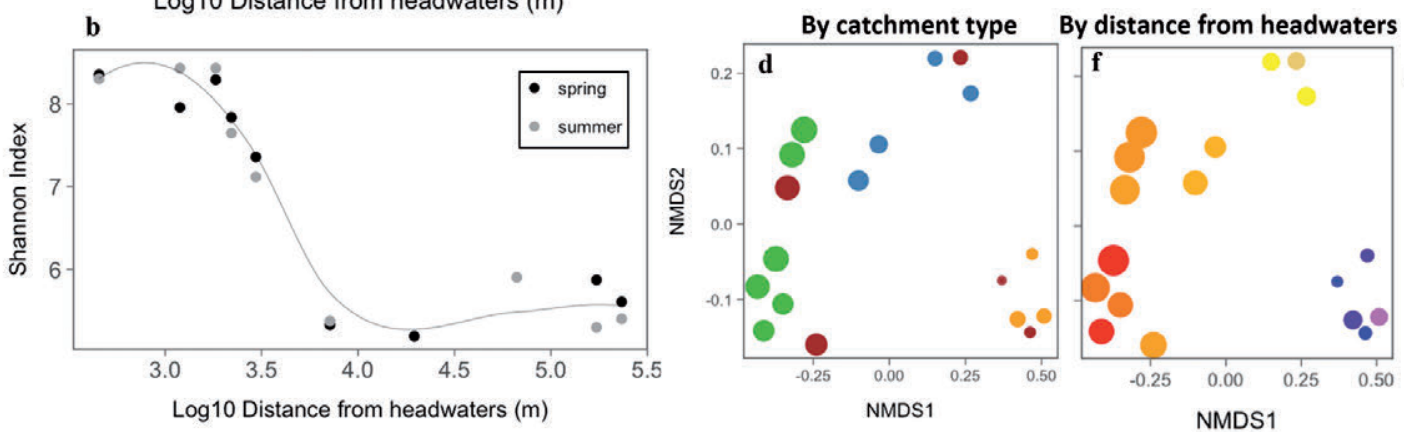

- 5000

- 7500

10000

12500

15000
17500

Catchment type

- Peatbog

- Clay soils

- Mixed forest

- Broad-leaf forest

$\log _{10}$ Upstream

distance(m)

5.0

4.5

4.0

3.5

3.0

Fig. 1. Change in OTU number (a) and taxonomic diversity (Shannon index, b) as a function of the measured distance between each sampling point and its headwaters. The lines are a best-fit smooth curve through the centre of the data calculated using weighted least squares. Each pair of dots represents two sampling points (spring, black; summer, grey) of the same site. c-f) Non-metric multidimensional scaling (NMDS) plots based on Bray-Curtis distances of taxonomic composition of all communities, color-coded by season (c), catchment type (d), region (e) and distance from the headwaters (f). The size of the dot is proportional the number of OTUs per community. 

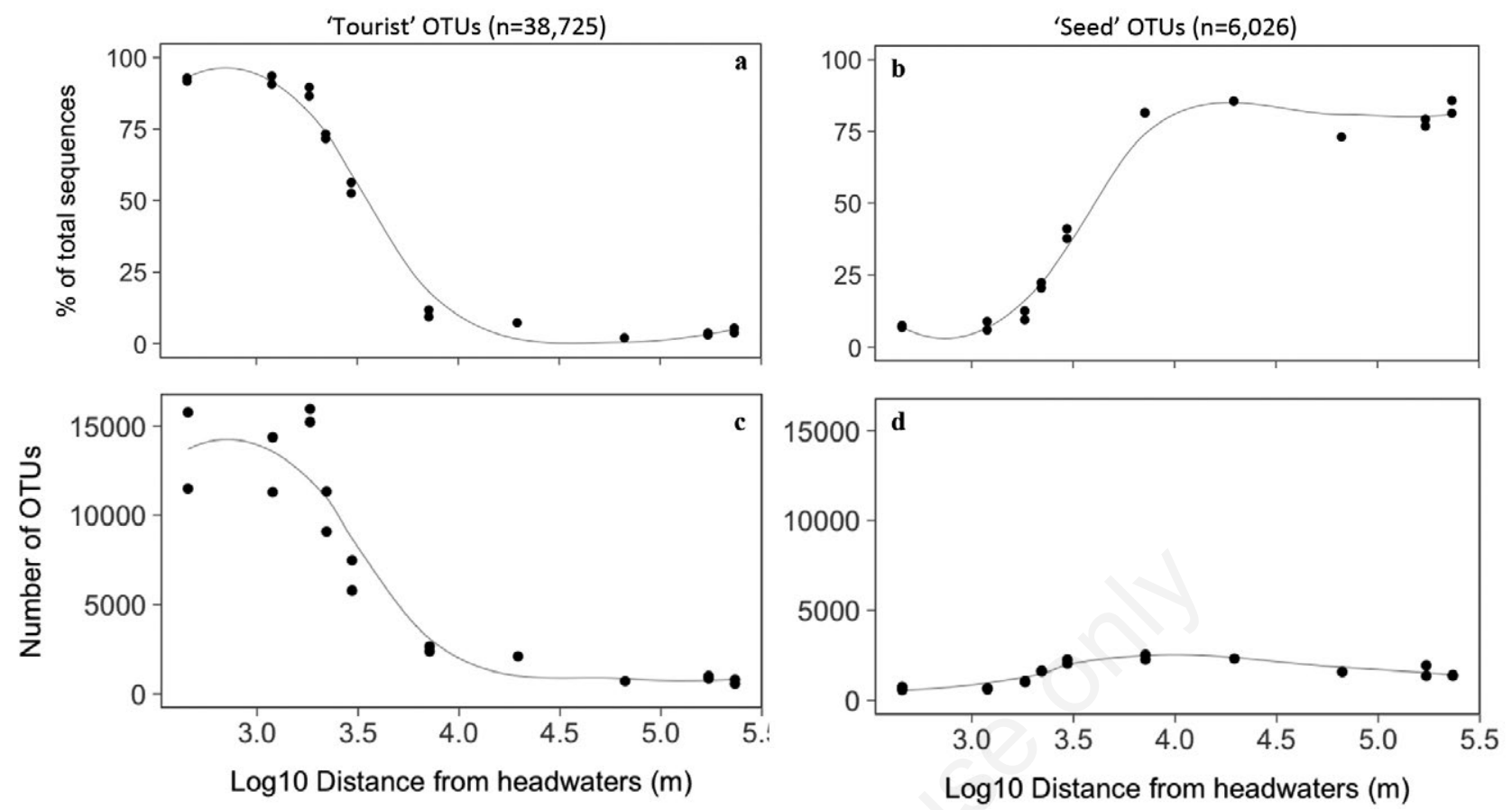

Fig. 2. Contribution of headwater OTUs (i.e., those OTUs detected in streams of order 0 and 1, see Methods) to total bacterial sequences $(\mathrm{a}, \mathrm{c})$ or OTUs $(\mathrm{c}, \mathrm{d})$ in aquatic communities as a function of the measured distance between each sampling point and its headwaters. Headwater OTUs were divided depending on their dynamics along the hydrologic continuum into 'Tourist' OTUs (OTUs whose abundances decreased towards rivers, a,c) and 'Seed' OTUs (OTUs whose abundances increased towards rivers, b,d). The lines are a bestfit smooth curve through the center of the data calculated using weighted least squares. Each pair of dots represents two sampling points (spring, summer) of the same site.

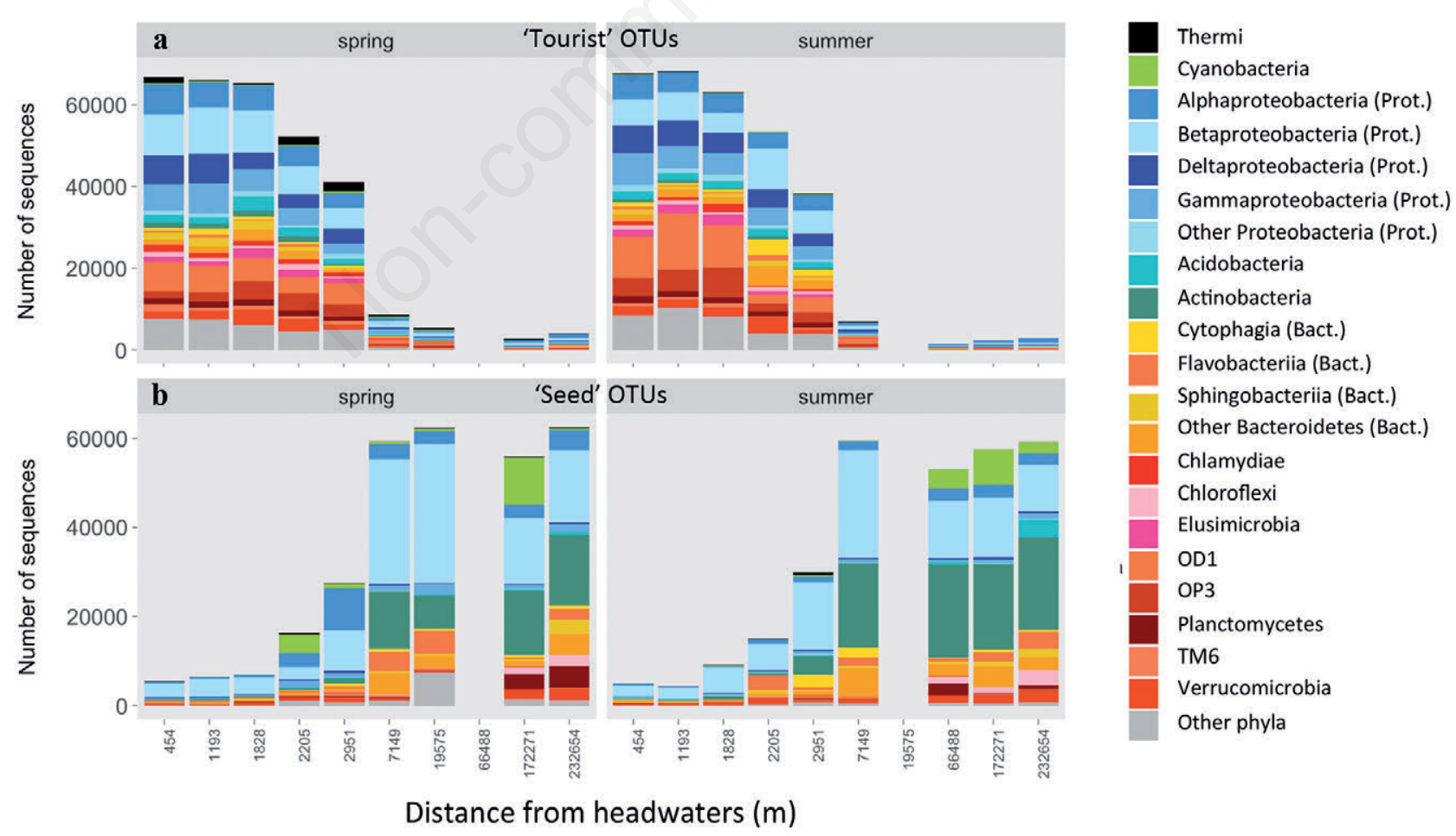

Fig. 3. Taxonomic composition of 'Seed' and 'Tourist' OTUs along a gradient of increasing distance from the headwaters. Data are shown separately for the two sampling times, and are presented as the sum of total sequences associated to each group. The classification was performed at the phylum level in most cases, but the Phyla Proteobacteria (Prot) and Bacteroidetes (Bact) were split into classes. 
It is important to note that these two groups of sites did not differ largely in the relative abundance of either 'Seeds' or 'Tourist' OTUs regardless of the differences in distance travelled (Figs. 2 and 4). Therefore, the observed changes in composition between $\mathrm{t} 1$ and $\mathrm{t} 2$, and between s1 and s2, must be due to changes on the group membership as a response to environmental factors and not only to direct hydrologic transport.

In order to assess so, using envfit analysis we explored the environmental variables that best explained the taxonomic differences between those two groups of sites (t1 $v s \mathrm{t} 2$ and $\mathrm{s} 1 v s \mathrm{~s} 2$ ) for each of the taxa pools (i.e., the smallest headwater streams in the case of communities dominated by 'Tourist' OTUs, and the largest rivers in the case of communities dominated by 'Seed' OTUs, Fig. 5). We observed that the compositional differences between the two groups of communities dominated by 'Tourist' OTUs ( $\mathrm{t} 1$ and $\mathrm{t} 2$ ) were associated with differences in the ratio of cDOM to DOC, which is a proxy of terrestrial influence, as well as in the proportions of the DOM fluorescent components $\mathrm{Cl}$ (associated to processed humic-like material) and C3 (freshly produced humic-like material, Fig. 5a). Conversely, variation between the two groups of 'Seed'-dominated communities in the largest rivers (s1 and s2) seemed to be due to differences in autochthonous processes, since chlorophyll $a$ concentration and $\%$ of $\mathrm{C} 5$ (associated to algal material) component appeared as the variables most clearly explaining the observed pattern, together with another humic-like component C2 (Fig. 5b). In general, the taxonomic differences between 'Seed' dominated communities were larger than those between communities dominated by 'Tourist' OTUs (see axes in Fig. 5).

Finally, we investigated differences between the metabolic profiles (assessed by the Biolog Ecoplates) of all communities (Fig. 6). Interestingly, we observed clear metabolic differences between communities dominated by 'Seed' and 'Tourist' OTUs, with the exception of one site that corresponded to the only river of order 6 , which grouped together with the headwater streams (Fig. 6a). These differences seemed mostly caused by variations in the use of four carbohydrates (D-Cellobiose, D-Lactose, Glucose, Erythritol), one carboxylic acid (2-Hydroxy Benzoic Acid) and one amino acid (L-Serine, Fig. 6a). In general, communities dominated by 'Seed' OTUs (i.e., large rivers) displayed higher capacity to respire L-Serine and Erythritol and lower capacity to use D-Cellobiose, DLactose and 2-Hydroxy Benzoic Acid than communities dominated by 'Tourist' OTUs. However, 'Tourist'-dominated communities showed larger metabolic differences between groups t 1 and 2 than 'Seed' communities, which showed less clear differentiation between s1 and s2 (Fig. 6a). Interestingly, we observed a gradient of increasing metabolic dissimilarity along a gradient of river order (Fig. 6b), suggesting that communities from the small headwater streams were more functionally similar to each other than communities located towards the lower end of the hydrologic continuum.
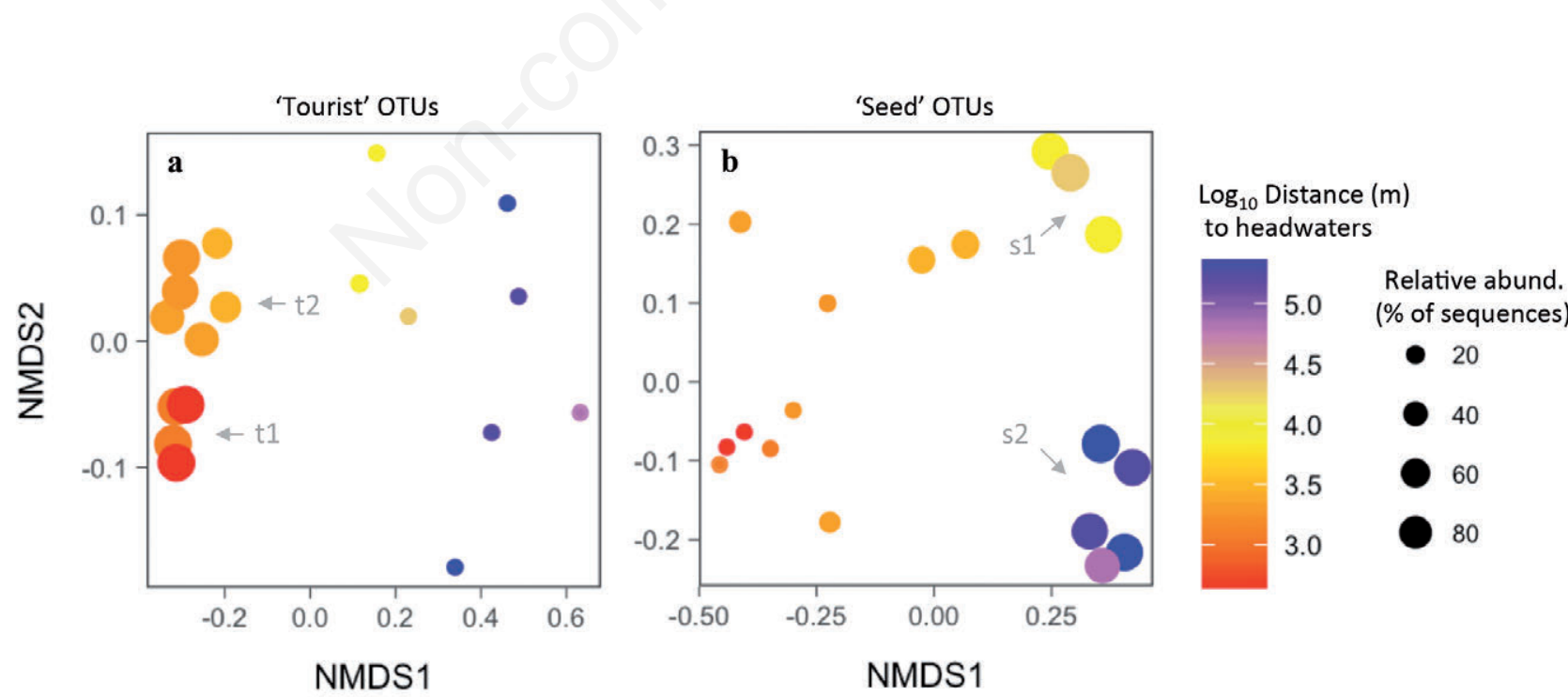

Fig. 4. Non-metric multidimensional scaling (NMDS) plots based on Bray-Curtis distances of taxonomic composition of both the 'Seeds' and 'Tourist' OTUs (see legend of Fig. 1). The color gradient indicates the distance between each sampling point and its headwaters, and the size of the dot is proportional to the relative contribution of either 'Seed' or 'Tourist' OTUs to each community' sequences. For each taxa pool, the two arrows indicate the two groups of communities where each type of OTU dominated (t1 and t2 in 'Tourist'dominated communities and s1 and s2 in 'Seed'-dominated communities), which were compared in order to understand the environmental drivers explaining the observed changes in composition but not in abundance (see Figs. 5 and 6). 


\section{DISCUSSION}

Our results show a sequential structuring of riverine bacterioplankton communities, which changed from highly diverse communities inhabiting the smallest headwater streams, towards assemblages with much lower tax-
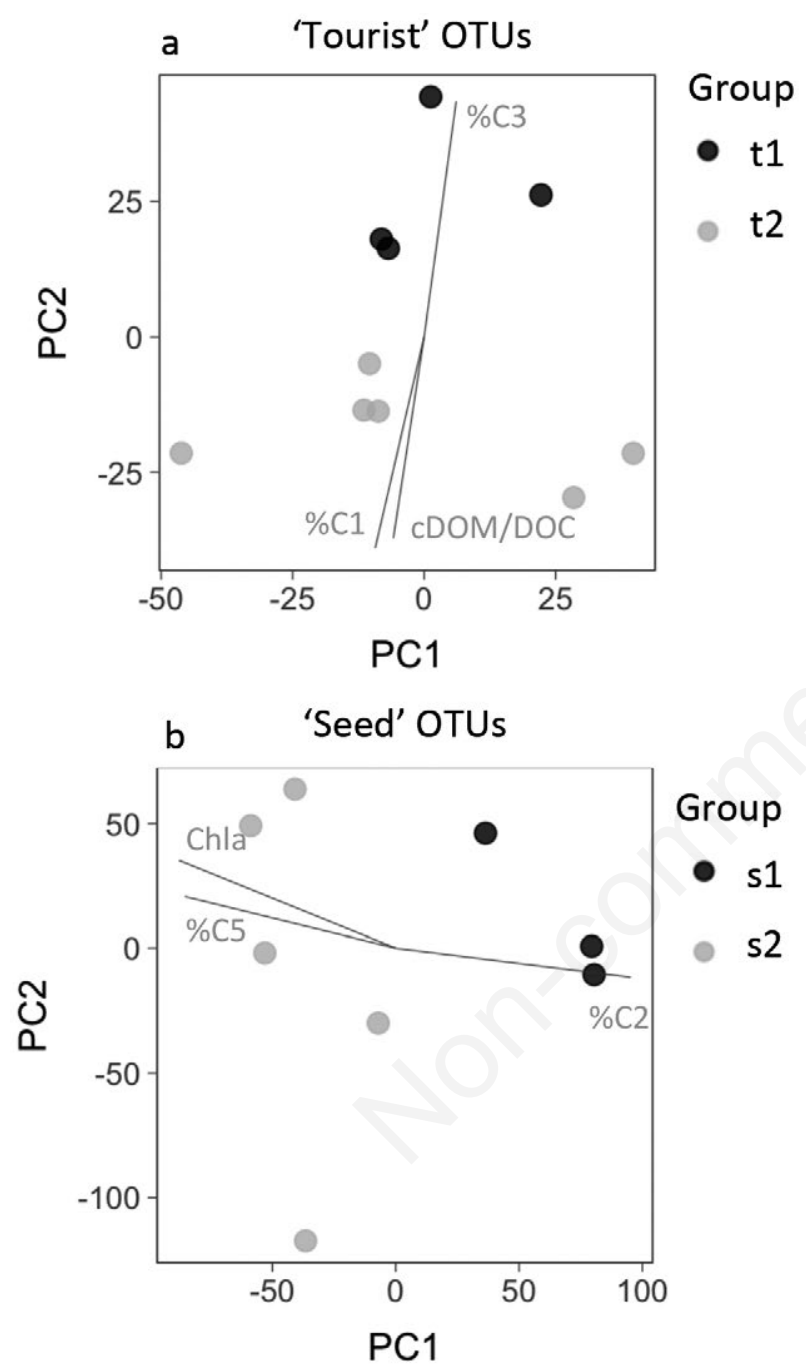

Fig. 5. PCA analysis of the communities where 'Seed' (a) and 'Tourist' (b) OTUs dominated, color-coded by the two groups of sites ( $\mathrm{t} 1$ and $\mathrm{t} 2$ in 'Tourist'-dominated communities and $\mathrm{s} 1$ and $\mathrm{s} 2$ in 'Seed'-dominated communities) observed in Fig. 4. The vectors show the environmental variables that best fitted the ordination space (using the R envfit funtion). The size of the vector is proportional to the strength of the correlation of each variable. $\mathrm{cDOM} / \mathrm{DOC}$, ratio of coloured dissolved organic matter to dissolved organic carbon concentration (a proxy of terrestrial inputs); Chla, Chlorophyll $a$ concentration; $\% \mathrm{C} 1, \% \mathrm{C} 2, \% \mathrm{C} 3, \% \mathrm{C} 5$, percentage contribution of the fluorescent parallel factor analysis components $\mathrm{C} 1,2,3$ and $\mathrm{C} 5$ to the total fluorescence. onomic richness and diversity inhabiting the largest rivers (Fig. $1, b)$. This pattern was also reflected by an organization of communities along a gradient of cumulative distance from the headwaters (i.e., travelled distance, Fig. 1f) that coincided with concomitant increases in river order (details not shown) and likely also water residence time, although the later was not directly measured. The fact that the observed structure was independent from the geographic location of the rivers, the sampling period, and the catchment type (Fig. 1), supports previous results showing a major role of hydrology and landscape connectivity in shaping the structure of freshwater bacterioplankton communities (Crump et al., 2007; Nelson et al., 2009; Niño-García et al., 2016a).

The high numbers of OTUs that we observed in the smallest headwater streams agrees with the notion that these systems act as integrators and dispersers of microbial diversity originating from the surrounding terrestrial landscape (Savio et al., 2015; Hauptmann et al., 2016; Ruiz-González et al., 2015a; Niño-García et al 2016a). Moreover, whereas the observed decrease in OTU richness suggests that many of these dispersed bacteria cannot thrive in the water, the reduction in taxonomic diversity further implies that there is a progressive dominance of taxa adapted to aquatic conditions, as previously reported (Savio et al., 2015; Niño-García et al 2016a). In order to check whether we could differentiate such contrasting dynamics within the dispersed taxa, we identified those OTUs present at the origin of the hydrologic continuum (i.e., streams of order 0 or 1) that i) decreased in abundance (i.e., 'Tourist' OTUs) or ii) increased in abundance ('Seed' OTUs) towards the largest rivers. Doing so, 58\% of all OTUs were categorized as 'Tourist', and only 9\% as 'Seeds' (Fig. 2), but together represented $92.4 \%$ of the total sequences in the dataset, suggesting that these simple criteria captured the most important community dynamics across the studied riverine ecosystems.

We observed pronounced decreases in OTU number and relative contribution of 'Tourist' taxa from the headwaters to the largest rivers. These two parameters ('Tourist' OTU richness and abundance, Fig. 2 a,c) showed a very similar pattern and indeed largely covaried $\left(\mathrm{R}^{2}=0.93, \mathrm{n}=18\right.$, $\mathrm{P}<0.0001$ ), suggesting that the observed abundance decrease of 'Tourist' OTUs was likely the result of dilution or removal of taxa that could not grow in the aquatic environment. The presence of typical soil or groundwater groups such as Acidobacteria, Deltaproteobacteria, Thermi, OD1, OP3, Verrucomicrobia within the 'Tourist' group supports the fact that their presence is largely due to advection from the catchment (Janssen et al., 2002; Lauber et al., 2009; Barberán et al., 2014; Hiller et al., 2015). Conversely, 'Seed' OTUs displayed pronounced increases in abundance along the hydrologic gradient that were not accompanied by comparable increases in the number of OTUs (Fig. 2 
b,d). This indicates that 'Seed' OTUs comprise a smaller pool of taxa, also present at the origin of the hydrologic continuum, which grow during transit through riverine networks and dominate downstream assemblages. Based on previous studies, it is likely that most of these 'Seeds' are actually washed from the surrounding soils, where they can persist at low abundances (Crump et al., 2012; RuizGonzález et al., 2015a), maybe dormant (Lennon and Jones 2011). Moreover, this distinction between 'Seeds' and 'Tourist' OTUs is in accordance with the partition of boreal lake bacterioplankton communities into 'core' (adapted to local conditions) and 'accidental' (linked to hydrologic transport) components based on their large-scale spatial dynamics (Niño-García et al., 2016b), and supports the notion that the main community assembly mechanisms change gradually along hydrologic gradients, shifting from communities strongly subjected to mass effects in the fast flowing headwater streams that are highly influenced by terrestrial inputs, to assemblages where local sorting of species will prevail (Lindström et al., 2006; Crump et al., 2007; Nelson et al., 2009; Ruiz-González et al.,, 2015; Niño-García et al., 2016a).

Interestingly, we observed that such increases and decreases in the abundances of 'Seed' and 'Tourist' OTUs, respectively, tended to stabilize beyond a travelled distance of ca. $7 \mathrm{~km}$ (Fig. $2 \mathrm{a}, \mathrm{b}$ ), corresponding to the rivers of order 4. This coincides with our own previous observation, in a different region of boreal Québec, that the proportion of sequences associated to the soil-derived taxa able to grow in the water increased progressively from the smallest streams until rivers of order 4 , beyond which their contribution to total sequences tended to plateau at ca. 70\% (Ruiz-González et al., 2015a). Since this stabilization corresponded approximately to a water residence time of 10 days, it was suggested that beyond 10 days hydrology no longer constrains the growth of taxa, and thus any further variations in community composition will be due to local selection driven by changes in aquatic physicochemical or biological conditions (Ruiz-González et al., 2015a; Niño-García et al., 2016a).

Accordingly, here we observed that although beyond 7 $\mathrm{km}$ the relative contribution of 'Seed' OTUs remained remarkably constant (around $80 \%$ of the community sequences), there were pronounced changes in taxonomic composition between those communities (likely due to changes in river physicochemical conditions), which showed two clear groups of sites corresponding to rivers of order 4 (s1) and rivers of order 6-7 (s2, Fig. 4b). Similarly,
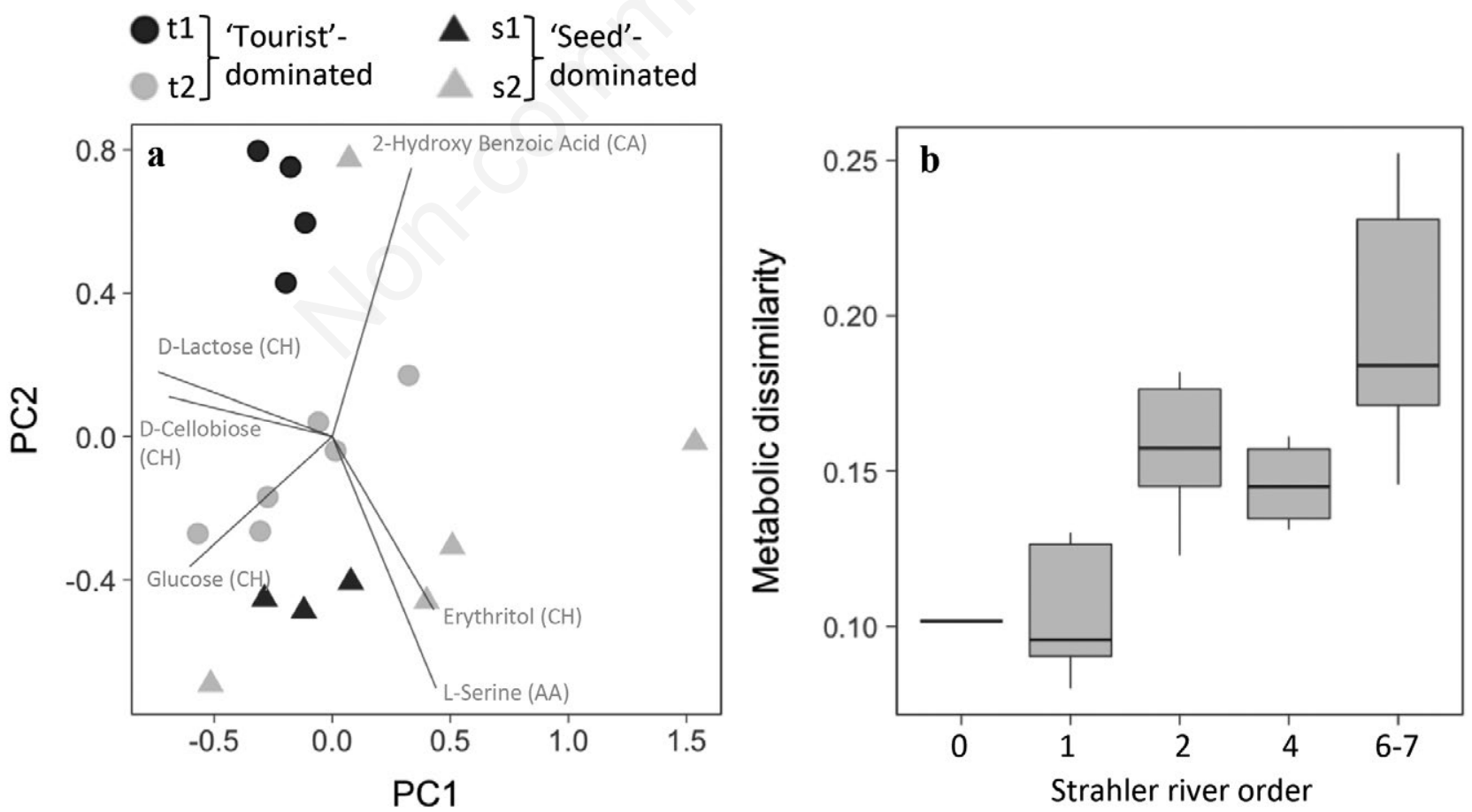

Fig. 6. a) PCA based on the metabolic profiles (Biolog Ecoplates) of all communities. Different shapes indicate whether 'Tourist' or 'Seed' OTUs dominated the communities (see arrows in Fig. 4). The vectors represent the carbon substrates that significantly explained the observed ordination; CA, carboxylic acid; $\mathrm{CH}$, crbohydrate; AA, amino acid. b) Increases in metabolic dissimilarity (assessed as Euclidean distance between samples characterized through Biolog profiles) as a function of Strahler river order. 
at the other end of the hydrologic gradient, communities dominated by 'Tourist' OTUs (i.e., those in the smallest headwater streams, order 0-2) also clustered into two different groups of sites ( $t 1$ and $t 2$, Fig. 4a). We expected that such compositional differences in community composition would be driven by different environmental factors at each extreme of the hydrologic gradient, since 'Tourist' taxa should mainly reflect the terrestrial environments where they originate, whereas 'Seed' taxa should be subjected to species selection driven by local aquatic environmental conditions. To assess so, we split the dataset into communities dominated by 'Tourist' or 'Seed' OTUs, respectively, and explored their variability in relation to all the measured variables (Fig. 5). Variables associated to DOM quality emerged as the most strongly related to the ordination in both cases, but they differed between 'Seed'- and 'Tourist'dominated communities (Fig. 5 a,b). For example, taxonomic differences between the two groups of communities dominated by 'Tourist' taxa in streams ( $\mathrm{t} 1$ and $\mathrm{t} 2$ ) seemed to be related to differences in the ratio of $\mathrm{CDOM}$ to DOC (a proxy of terrestrial influence; Lapierre and del Giorgio, 2014) or in the proportion of the DOM fluorescent components $\mathrm{C} 1$ (associated to processed humic-like material) and C3 (freshly produced humic-like material, Fig. 5a). Although we could not see a clear clustering of communities based on the different catchment types shown in Tab. 1, not even for the headwater assemblages (Fig. 1), this observation supports the hypothesis that headwater streams reflect to some extent a signature of the surrounding catchment, which in these same rivers had been shown to provide DOC of different quality (Berggren and del Giorgio, 2015). In this regard, Niño-García et al., (2016) observed a strong structuring effect of $\mathrm{pH}$ on boreal bacterioplankton communities, but attributed this to a regional landscape signature on the immigrant pool rather than to species sorting due to $\mathrm{pH}$ itself. Similarly, Lear et al., (2013) found that the biogeography of stream biofilm bacterial communities was related to variability in catchment land use, supporting a strong influence of the surrounding terrestrial environment in systems with high connectivity with land.

On the other hand, taxonomic variation across 'Seed' communities in the largest rivers appeared to be driven by differences in local aquatic processes, since chlorophyll $a$ concentration and \% $\%$ (associated to freshly-produced algal material) component appeared as significant environmental variables (Fig. 5b) and varied largely between the two groups of sites s1 and s2. Chlorophyll concentrations were higher in larger rivers independently of the surrounding catchment type (details not shown), which agrees with the expected pattern of downstream increase in planktonic chlorophyll, associated with longer residence times and increases in nutrients (Sabater et al., 2008), and this likely triggered the growth of bacterial taxa adapted to more productive conditions in $\mathrm{s} 2$ than in s1 sites. In any case, the observation that all these 'Seed' taxa, regardless of their taxonomic identity, were detected in the headwater streams supports that landscapes in these regions contain a reservoir of diversity able to colonize aquatic ecosystems if the water residence time is long enough (Ruiz-González et al., 2015a).

Along this same line, it is remarkable to note that the studied streams and rivers were not physically connected; all of them were located in different watersheds in two different geographic regions, separated by more than $350 \mathrm{~km}$. Therefore, the fact that those OTUs present across the sampled headwaters included taxa that would become dominant in all of the larger rivers suggests that there must be a large regional pool of taxa from which aquatic communities recruit, which is not subjected to dispersal limitation. Interestingly, this idea of the landscape reservoir of aquatic diversity has been proposed at scales much larger than the present study (Niño-García et al., 2016a; Ruiz-González et al., 2015a, 2017) and is in accordance with the seed-bank concept, a standing reservoir of dormant microbes (i.e., microbes in a reversible state of very low metabolic activity) that persist at low abundances but that can grow and become abundant upon changes in environmental conditions (Lennon and Jones, 2011). This further highlights that microbial seed banks may transcend ecosystem types, such that viable microbes can persist under unfavourable conditions in some ecosystems until they are transported to a suitable environment (Sjöstedt et al., 2012; Lee et al., 2013; Comte et al., 2014; Ruiz-González et al., 2015a, 2017). Soils in particular are known to contain a large fraction of dormant cells (Lennon and Jones, 2011), a fraction of which seems able to resuscitate and grow upon advection to the water. In any case, this fact challenges our current ecosystem-specific approaches to study bacterial communities, and highlights that it will not be possible to understand how communities are assembled or structured without considering at least the most important potential sources of microbial dispersal.

Finally, the fact that such a large fraction of the diversity may represent taxa that are not adapted to local conditions has important implications for our understanding of community functioning. This agrees with recent evidence suggesting that a potentially large fraction of the microbial diversity detected in biogeographic studies can be either dead, in a dormant state, or present as extracellular DNA (Lennon and Jones, 2011; Blagodatskaya and Kuzyakov, 2013; Carini et al., 2016). In this regard, exploring the dynamics of different taxa across ca. 200 lakes Niño-García et al. (2016a) revealed that the presence of most rare bacterial taxa was due to hydrologic transport from the associated networks, and thus these taxa likely represent a species pool that is not contributing to the functioning of communities but that is detected in all sequencing studies. For these reasons, we expected to find 
a clearer link between taxonomy and the metabolic profiles of the studied communities when considering separately the pool of 'Seed' and 'Tourist' taxa than when considering the whole communities, and in addition, we expected to find a stronger correlation between taxonomy and function for 'Seeds' than for 'Tourist' OTUs. However, this was not the case: changes in taxonomic composition were weakly but significantly correlated to changes in metabolic profiles in all three cases, i.e., when the whole community was considered (Mantel $\mathrm{R}=0.189$, $\mathrm{P}<0.05$ ), or when considering 'Seeds' (Mantel $\mathrm{R}=0.201$, $\mathrm{P}<0.05$ ) or 'Tourist' OTUs (Mantel $\mathrm{R}=0.215, \mathrm{P}<0.05$ ) separately, and the strength of the correlation was very similar in all cases. This may be due to the fact that the Biolog Ecoplates used for assessing the metabolic profiles do not measure in situ activity levels but rather a functional potential, because it is a method that relies on the growth of microorganisms on the selected substrates during an incubation of several days (Garland et al., 2001). Thus, the microorganisms that will grow on the plates may be rare in the original communities (Smalla et al., 1998), particularly in headwaters where taxa do not have the time to attain high abundances due to the fast hydraulics. However, we did observe clear metabolic differences between communities dominated by 'Seeds' and 'Tourist' OTUs (Fig. 6a), suggesting that the functional structure or potential of these communities actually differs. Interestingly, moreover, whereas communities from the headwater streams were functionally more similar to each other, assemblages in large rivers seemed much more heterogeneous metabolically (Fig. 6b). This agrees with a previous study where we analyzed the metabolic profiles of ca. 300 freshwater bacterioplankton assemblages and found that communities from systems more tightly connected to the land were more similar metabolically than communities from larger lakes or rivers, and this increase in metabolic dissimilarity followed a gradient of increasing concentrations of autochthonously produced organic carbon (Ruiz-

Tab. 2. ANOSIM R values indicating significant differences in Biolog metabolic profiles between different categories. All R values for Euclidean distance matrices were calculated using 9999 permutations. Significance codes for R values Community group refers to the 4 groups of communities where either 'Seed' or 'Tourist' OTUs dominated (see arrows in Fig. 4).

\begin{tabular}{lc} 
& ANOSIM R values \\
By season & $\mathrm{ns}$ \\
By region & $\mathrm{ns}$ \\
\hline By catchment type & $038^{* *}$ \\
By river order & $0.19^{*}$ \\
\hline By community group & $0.16^{*}$ \\
\hline ns, not significant; $* * \mathrm{P}<0.0001 ; * \mathrm{P}<0.05$
\end{tabular}

González et al., 2015b). This pattern may suggest that as systems depart from land, the structural diversification of the available DOM pool due to local processes (Kothawala et al., 2014) causes a functional diversification, whereas terrestrial DOM is more homogeneous or simply the pool of headwater taxa that can grow on the substrates is more restricted that in true aquatic communities. It is important to note that this diversification of function along the aquatic flow path does not come in parallel to a taxonomic diversification; in fact, there was an overall decline in OTU richness along this same continuum. In a previous study on these same rivers (Berggren and del Giorgio, 2015) the type of catchment (mainly forest headwater versus peat influenced) was shown to be the strongest determinant of multiple facets of bacterial metabolism (e.g., biomass production, respiration, growth efficiency) presumably driven by watershed-scale differences in DOC quality and quantity. Indeed, we found that catchment type was the variable explaining most of the metabolic differences between communities (Tab. 2). However, we also found weaker but significant differences based on the four types of communities (Tab. 2), suggesting that the taxonomic composition also plays a role in determining the metabolic potential of communities in these heterogeneous rivers.

\section{CONCLUSIONS}

Overall, our results show that bacterioplankton communities from lotic systems may comprise varying proportions of taxa adapted and not adapted to live in aquatic conditions, and this balance is largely driven by the time that bacteria have spent in a given parcel of water. Since the assembly mechanisms of both components differ, the environmental variables explaining shifts in taxonomic composition also change between the two pools of taxa, and whereas variables related with terrestrial inputs from the catchment explained differences in communities dominated by 'Tourist' OTUs, varying concentrations of chlorophyll and the associated DOC appeared to be related to compositional changes in 'Seed'-dominated communities. Finally, we found that the metabolic potential associated to these contrasting taxa pools also differed. All this highlights the need to consider the landscape connectivity between assemblages and the resulting dynamics of specific populations in order to fully understand the assembly mechanisms and environmental factors shaping these flowing communities.

\section{ACKNOWLEDGEMENTS}

We thank the whole CarBBAS team for their contribution to the field and laboratory components of this re- 
search. In particular, we thank J.F. Lapierre for providing the PARAFAC data. This study is part of the program of the Carbon Biogeochemistry in Boreal Aquatic Systems (CarBBAS) Industrial Research Chair, co-funded by the Natural Science and Engineering Research Council of Canada (NSERC) and Hydro-Quebec. We acknowledge funding from FRQNT, NSERC, and the Canada Research Chairs program. CRG benefited of a Juan de la Cierva post-doctoral fellowship from the Spanish Ministry of Industry, Economy and Competitivity.

\section{REFERENCES}

Barberán A, Ramirez KS, Left JW, Bradford MA, Wall DH, Fierer N, 2014. Why are some microbes more ubiquitous than others? Predicting the habitat breadth of soil bacteria. Ecol. Lett. 17:794-802.

Berggren M, del Giorgio PA, 2015. Distinct patterns of microbial metabolism associated to riverine dissolved organic carbon of different source and quality. J. Geophys. Res. Biogeo.120:989-999.

Besemer K, Singer G, Quince C, Bertuzzo E, Sloan W, Battin TJ 2013. Headwaters are critical reservoirs of microbial diversity for fluvial networks. P. Roy. Soc. B. 280:20131760.

Besemer K, Peter H, Logue JB, Langenheder S, Lindstrom ES, Tranvik LJ, Battin TJ, 2012. Unraveling assembly of stream biofilm communities. ISME J. 6:1459-1468.

Blagodatskaya E, Kuzyakov Y, 2013. Active microorganisms in soil: Critical review of estimation criteria and approaches. Soil Biol. Biochem. 67:192-211.

Caporaso JG, Lauber CL, Walters WA, Berg-Lyons D, Huntley J, Fierer N, et al. 2012. Ultra-high-throughput microbial community analysis on the Illumina HiSeq and MiSeq platforms. ISME J. 6:1621-1624.

Caporaso JG, Kuczynski J, Stombaugh J, Bittinger K, Bushman FD, Costello EK, et al. 2010. QIIME allows analysis of high-throughput community sequencing data. Nat. Methods 7:335-336.

Carini P, Marsden PJ, Leff JW, Morgan EE, Strickland MS, Fierer N, 2016. Relic DNA is abundant in soil and obscures estimates of soil microbial diversity. Nat. Microbiol. 2:16242.

Clarke KR, 1993. Non-parametric multivariate analysis of changes in community structure. Australian J. Ecol. 18:117-143.

Comte J, Lindström ES, Eiler A, Langenheder S, 2014. Can marine bacteria be recruited from freshwater sources and the air? ISME J. 8:2423-2430.

Crump BC, Amaral-Zettler LA, Kling GW, 2012. Microbial diversity in arctic freshwaters is structured by inoculation of microbes from soils. ISME J. 6:1629-1639.

Crump BC, Adams HE, Hobbie JE, Kling GW, 2007. Biogeography of bacterioplankton in lakes and streams of an Arctic tundra catchment. Ecology 88:1365-1378.

Edgar RC, 2010. Search and clustering orders of magnitude faster than BLAST. Bioinformatics 26:2460-2461.

Garland JL, Mills AL, Young JS, 2001. Relative effectiveness of kinetic analysis vs single point readings for classifying environmental samples based on community-level physiological profiles CLPP. Soil Biol. Biochem. 33:1059-1066.
Hiller KA, Foreman KH, Weisman D, Bowen JL, 2015. Permeable reactive barriers designed to mitigate eutrophication alter bacterial community composition and aquifer redox conditions. Appl. Environ. Microbiol. 81:7114-7124.

Janssen PH, Yates PS, Grinton BE, Taylor PM, Sait M, 2002. Improved culturability of soil bacteria and isolation in pure culture of novel members of the divisions Acidobacteria, Actinobacteria, Proteobacteria, and Verrucomicrobia. Appl. Environ. Microbiol. 68:2391-2396.

Kothawala DN, Stedmon CA, Müller RA, Weyhenmeyer GA, Köhler SJ, Tranvik L, 2014. Controls of dissolved organic matter quality:evidence from a large-scale boreal lake survey. Glob. Change Biol. 20:1101-1114.

Lapierre JF, del Giorgio PA, 2014. Partial coupling and differential regulation of biologically and photochemically labile dissolved organic carbon across boreal aquatic networks. Biogeosciences 11:5969-5985.

Lauber CL, Hamady M, Knight R, Fierer N, 2009. Pyrosequencing-based assessment of soil $\mathrm{pH}$ as a predictor of soil bacterial community structure at the continental scale. Appl. Environ. Microbiol. 75:5111-5120.

Lear G, Washington V, Neale M, Case B, Buckley H, Lewis G, 2013. The biogeography of stream bacteria. Global Ecol. Biogeogr. 22:544-554.

Lee JE, Buckley HL, Etienne RS, Lear G, 2013. Both species sorting and neutral processes drive assembly of bacterial communities in aquatic microcosms. FEMS Microbiol. Ecol. 86:288-302.

Lennon JT, Jones SE, 2011. Microbial seed banks:the ecological and evolutionary implications of dormancy. Nat. Rev. Microbiol. 119:119-130.

Lindström ES, Bergström AK, 2004. Influence of inlet bacteria on bacterioplankton assemblage composition in lakes of different hydraulic retention time. Limnol. Oceanogr. 49:125-136.

Lindström ES, Forslund M, Algesten G, Bergström A, 2006. External control of bacterial community structure in lakes. Limnol. Oceanogr. 51:339-342.

Logares R, Lindström ES, Langenheder S, Logue JB, Paterson H, Laybourn-Parry J, et al. 2013. Biogeography of bacterial communities exposed to progressive long-term environmental change. ISME J. 7:937-948.

Magoc T, Salzberg S, 2011. FLASH: Fast length adjustment of short reads to improve genome assemblies. Bioinformatics 27:2957-2963.

Nelson CE, Sadro S, Melack JM, 2009. Contrasting the influences of stream inputs and landscape position on bacterioplankton community structure and dissolved organic matter composition in high-elevation lake chains. Limnol. Oceanogr. 54:1292-1305.

Newton RJ, Jones SE, Eiler A, McMahon KD, Bertilsson S, 2011. A guide to the natural history of freshwater lake bacteria. Microbiol. Mol. Biol. Res. 75:14-49.

Niño-García JP, Ruiz-González C, del Giorgio PA, 2016a. Interactions between hydrology and water chemistry shape bacterioplankton biogeography across boreal freshwater networks. ISME J. 10:1755-1766.

Niño-García JP, Ruiz-González C, del Giorgio PA, 2016b. Landscape-scale spatial abundance distributions discriminate core from random components of boreal lake bacterioplankton. Ecol. Lett. 19:1506-1515. 
Pruesse E, Quast C, Knittel K, Fuchs BM, Ludwig W, Peplies J, et al. 2007. SILVA:a comprehensive online resource for quality checked and aligned ribosomal RNA sequence data compatible with ARB. Nucleic Acids Res. 35:71887196.

Ruiz-González C, Niño-García JP, del Giorgio PA, 2015a. Terrestrial origin of bacterial communities in complex boreal freshwater networks. Ecol. Lett. 18:1198-1206.

Ruiz-González C, Niño-García JP, Lapierre JF, del Giorgio PA, $2015 \mathrm{~b}$. The quality of organic matter shapes the functional biogeography of bacterioplankton across boreal freshwater ecosystems. Global Ecol. Biogeogr. 11:11-12.

Ruiz-González C, Niño-García JP, Kembel SW, del Giorgio PA, 2017. Identifying the core seed bank of a complex boreal bacterial metacommunity. ISME J. 11:2012-2021.

Savio D, Sinclair L, Ijaz UZ, et al., 2015. Bacterial diversity along a $2600 \mathrm{~km}$ river continuum. Environ. Microbiol. 17:4994-5007.

Sjöstedt J, Koch-Schmidt P, Pontarp M, Canbäck B, Tunlid A, Lundberg P, et al. 2012. Recruitment of members from the rare biosphere of marine bacterioplankton communities after an environmental disturbance. Appl. Environ. Microbiol. 78:1361-1369.

Smalla K, Watchtendorf U, Heuer H, Liu W, Forney L, 1998. Analysis of BIOLOG GN substrate utilization patterns by microbial communities. Appl. Environ. Microbiol. 64:1220-1225. Souffreau C, Van der Gucht K, van Gremberghe I, Kosten S, Lacerot G, Lobão LM, et al., 2015. Environmental rather than spatial factors structure bacterioplankton communities in shallow lakes along a $>6000 \mathrm{~km}$ latitudinal gradient in South America. Environ. Microbiol. 17:2336-2351.

Stubbins A, Lapierre JF, Berggren M, Prairie YT, Dittmar T, del Giorgio PA, 2014. What's in an EEM? Molecular signatures associated with dissolved organic fluorescence in boreal Canada. Envir. Sci. Tech. 48:10598-10606.

Wang Q, Garrity GM, Tiedje JM, Cole JR, 2007. Naïve bayesian classifier for rapid assignment of rRNA sequences into the new bacterial taxonomy. Appl. Environ. Microbiol. 73:5261-5267.

Wilhelm L, Besemer K, Fragner L, Peter H, Weckwerth W, Battin TJ, 2015. Altitudinal patterns of diversity and functional traits of metabolically active microorganisms in stream biofilms. ISME J. 9:2454-2464. 